\title{
SECTION III
}

CHEMICAL ABUNDANCES IN PLANETARY NEBULAE 
Manuel Peimbert and Silvia Torres-Peimbert

Instituto de Astronomía, Universidad Nacional Autónoma de México

Abstract. The general properties of PN of Type I are reviewed. A list of 29 PN of Type I is presented, most of them are bipolar. Their bipolar nature might be a direct consequence of the large masses and angular momenta of their progenitor stars. PN of Type I are He and $\mathrm{N}$ rich, their observed chemical abundances are compared with theoretical predictions. A group of Type I PN candidates is presented.

\section{INTRODUCTION}

PN of Type I were defined by Peimbert (1978) based on a handful of objects; it is the purpose of this review to study if PN of Type I form a coherent group with a unique set of physical properties. For example we want to know if they correspond to a given range of precursor masses. If that were the case we would be able to use them to test predictions based on stellar evolution theory (e.g. Renzini and Voli 1981) related to the chemical evolution of the surface layers. Moreover we would be able to use them for the study of the chemical evolution of galaxies (e.g. Serrano and Peimbert 1981).

\section{DEFINITION}

\section{Primary Criterion}

Peimbert (1978; see also Peimbert and Serrano 1980; hereinafter PS) defined $\mathrm{PN}$ of Type I as those objects with $\mathrm{N}(\mathrm{He}) / \mathrm{N}(\mathrm{H}) \geqslant 0.14$ or $\log \mathrm{N} / 0 \geqslant$ 0.00 . In past photographic work weak lines were systematically overestimated, this effect yields higher He/H ratios and higher electron temperatures which in turn produce spuriously large $\mathrm{N}^{+} / 0^{+}$and $\mathrm{N} / 0$ ratios. The number of photoelectric observations is increasing and with it the quality of the abundance determinations, therefore we decided to relax the definition to include those objects with $\mathrm{N}(\mathrm{He}) / \mathrm{N}(\mathrm{H}) \geqslant 0.125$ and $\log$ $\mathrm{N} / 0 \geqslant-0.3$ since we consider these objects to belong to a physical group of He and $\mathrm{N}$ rich objects. 
TABLE 1

Planetary Nebulae of Type I

\begin{tabular}{|c|c|c|c|c|c|c|c|c|}
\hline & bject & $\mathrm{He} / \mathrm{H}$ & $\log (N / 0)$ & $\begin{array}{c}\log (0 / H) \\
+12\end{array}$ & $\log (\mathrm{c} / 0)$ & $\begin{array}{l}\text { Source } \\
\text { Abund. }\end{array}$ & $\begin{array}{l}\text { Morph. } \\
\text { Kinem. }\end{array}$ & Source \\
\hline$\overline{\text { NGC }}$ & 650 & $0.130:$ & -0.37 & 8.87 & +0.31 & 1,2 & FBV & 19,20 \\
\hline NGC & 2346 & 0.130 & -0.37 & 8.63 & ... & 1,2 & FB & 18 \\
\hline NGC & $2371-2$ & 0.122 & -0.24 & 8.83 & +0.29 & 1,2 & FB & ... \\
\hline & 2440 & 0.112 & +0.21 & 8.87 & $+0.17^{a}$ & 1 & FB & 18,20 \\
\hline NGE & $\angle 440$ & 0.117 & +0.33 & 8.55 & -0.03 & 3 & ... & ... \\
\hline NGC & 2452 & 0.111 & +0.08 & 8.59 & . & 2 & FB & .. \\
\hline NGC & 2474-5 & ... & +0.08 & $\ldots$ & ... & 4 & FB & 21 \\
\hline NGC & 2818 & 0.143 & -0.06 & 8.69 & $+0.47^{a}$ & 1,5 & FB & $\cdots$ \\
\hline NGC & 3132 & 0.127 & -0.34 & 8.78 & .. & 5 & $S$ & ... \\
\hline NGC & 5189 & ... & ... & $\ldots$ & $\ldots$ & 6 & FB & 22 \\
\hline NGC & 5315 & 0.125 & -0.13 & 8.71 & ... & 5 & $S$ & .. \\
\hline NGC & 6302 & 0.186 & -0.20 & 8.70 & .. & 7 & FBV & $23,24,25$ \\
\hline & & 0.182 & +0.22 & 8.70 & -0.70 & 8 & $\cdots$ & $\cdots$ \\
\hline NGC & 6445 & 0.228 & -0.02 & . . & . & 9 & FB & ... \\
\hline NGC & 6537 & $>0.140$ & 0.00 & ... & ... & 10 & FB & 26 \\
\hline NGC & 6629 & $0.143:$ & ... & ... & ... & 11 & $S$ & 27 \\
\hline NGC & 6741 & 0.136 & -0.13 & ... & ... & 12,13 & $\mathrm{FB}$ & .. \\
\hline NGC & 6751 & 0.118 & -0.23 & 8.57 & $\ldots$ & 2,11 & F & ... \\
\hline NGC & 6778 & 0.155 & +0.03 & 8.48 & +0.53 & 2,11 & FB & ... \\
\hline NGC & 6853 & 0.133 & -0.26 & 8.78 & -0.15 & 13,14 & FBV & 28 \\
\hline NGC & 6894 & ... & $-0.02:$ & $\cdots$ & $\cdots$ & 4 & $S$ & 27 \\
\hline NGC & 7008 & 0.140 & 0.00 & $\ldots$ & $\ldots$ & 10,11 & $\mathrm{~S}$ & 27 \\
\hline IC & 4406 & 0.141 & -0.32 & $\cdots$ & .. & 4 & $\mathrm{~F}$ & ... \\
\hline $\mathrm{Hu}$ & $1-2$ & 0.152 & $+0.21:$ & 8.22: & $+0.27:$ & 1,2 & FBV & 29 \\
\hline $\mathbf{M}$ & $1-8$ & 0.127 & -0.37 & 8.81 & $+0.55^{a}$ & 1 & C & ... \\
\hline M & $2-55$ & 0.157 & -0.19 & $\ldots$ & . & $4,15,16$ & F & ... \\
\hline $\mathbf{M}$ & $3-3$ & 0.123 & +0.17 & 8.69 & $+0.58 \mathrm{a}$ & 1 & C & $\ldots$ \\
\hline Me & $2-2$ & 0.154 & -0.05 & $8.30:$ & $+0.52^{a}$ & 1,17 & $\mathrm{C}$ & ... \\
\hline Mz & 3 & $0.18:$ & $+0.1:$ & ... & . & 18 & FB & 30 \\
\hline $\mathrm{PB}$ & 6 & 0.185 & +0.17 & $8.38:$ & ... & 5 & $\mathrm{~F}$ & ... \\
\hline CRL & 618 & $\ldots$ & -0.10 & $\ldots$ & $\ldots$ & 18 & FBV & 31 \\
\hline
\end{tabular}

Notes to Table 1: ${ }^{a} \mathrm{C}^{++} / 0^{++}$ratio based on $\lambda 4267$ of C II; F filamentary; B bipolar, binebulous, biaxial, hourglass; V bipolar velocity field; S relatively smooth; C compact; 1 Peimbert and Torres-Peimbert 1982; 2 Aller and Czyzak 1982; 3 Shields et al. 1981; 4 Kaler 1982a; 5 TorresPeimbert and Peimbert 1977; 6 Worswick 1980; 7 Aller and Czyzak 1978; 8 Aller et al. 1981; 9 Aller et al. 1973; 10,11,12,13 Kaler 1982b,1980, 1978, 1979; 14 Pottasch 1981; 15 Sabbadin and Hamzaouglu 1981a; 16 this paper; 17 Barker 1978; 18 Calvet and Peimbert 1982; 19 Sabbadín and Hamzaouglu 1981b; 20 Minkowski 1964; 21 Phillips and Reay 1977; 22 Westerlund and Henize 1967; 23 Meaburn and Walsh 1980; 24 Barral et al. 1982; 25 Rodríguez and Morán 1982; 26 Felli and Perinotto 1979; 27 Curtis 1918; 28 Goudis et al. 1978; 29 Torres-Peimbert et al. 1982 ; 30 Cohen et al. 1978; 31 Carsenty and Solf 1982. 
Secondary Criteria

Most He and $\mathrm{N}$ rich $\mathrm{PN}$ show a very pronounced filamentary structure, and very strong forbidden lines from [0 I], [N I], [S II] up to [Ne V]. These objects comprise a subset of almost half of the objects classified by Greig (1971) as B nebulae (binebulous or filamentary). Greig (1972) found from kinematical properties that $B$ nebulae were population $I$ while A (annular) and C (centric) were of population II.

\section{CHEMICAL ABUNDANCES}

In Table 1 we present a list of He and $\mathrm{N}$ rich objects selected mainly from photoelectric observations. The estimated errors are smaller than 0.01 in $\mathrm{He} / \mathrm{H}$ and smaller than 0.2 in $\log \mathrm{N} / 0$ and in $\log 0 / \mathrm{H}$. When photo electric data are used from two different sources the average value is given. A previous compilation of PN of Types I and I-II by PS included 19 objects, of those 14 are presented in Table 1 and five have been deleted, the reasons are the following: $M 2-27$ and $\mathrm{Cn} 2-1$ belong to the bulge and could have been formed in a He and $\mathrm{N}$ rich medium, for NGC 7293 Peimbert and Torres-Peimbert (1982; hereinafter PTP) obtained $\mathrm{He} / \mathrm{H}=0.123$ and $\log \mathrm{N} / 0=-0.43$ and for $\mathrm{M} 1-80,0.095$ and -0.48 , while Aller and Czyzak (1982) for NGC 6309 obtained 0.120 and -0.91 .

Several objects deserve special attention: NGC 650 shows $\mathrm{N} \mathrm{He}^{+}+$ $\left.\mathrm{He}{ }^{++}\right) / \mathrm{N}\left(\mathrm{H}^{+}\right)=0.112$ and $\mathrm{S}^{+} / \mathrm{S}=0.14$ which is the largest value for the objects observed by PTP, by assuming that $\mathrm{He}^{\circ} / \mathrm{He}=\mathrm{S}^{+} / \mathrm{S}$ the value in Table 1 was derived. NGC 650 is the only object in Table 1 corrected for the presence of $\mathrm{He}^{\circ}$ inside the H II zone. For NGC 2440 and NGC 6302 we present two sets of data, the first is based on optical observations alone while the second includes IUE observations. The optical determina tions are based on the assumption that $\mathrm{N}^{+} / 0^{+}=\mathrm{N} / 0$, the UV observations include $\mathrm{N}^{++}, \mathrm{N}^{+3}$ and $\mathrm{N}^{+4}$. The UV results for objects of high degree of ionization yield in general somewhat larger $\mathrm{N}$ values than those derived optically (e.g. Peimbert 1981).

In Figure 1 we show a plot of $\mathrm{He} / \mathrm{H}$ versus $\mathrm{N} / \mathrm{O}$ where the shaded area corresponds to PN of Type II; in this area the objects with lower He and $N$ values are located in the outer regions of our galaxy, while the objects with higher He and $\mathrm{N}$ values are located towards the center of our galaxy (PS). The He and $\mathrm{N}$ rich objects are represented by filled circles and do not show any tendency related with galactic position, which implies that most of the excess He and $\mathrm{N}$ was produced by them (see also PS). In Figure 1 we also show the tracks predicted by Renzini and Voli (1981) for intermediate mass stars, most of the objects fall in the region for $2>\alpha>1$ and $3 \lesssim M / M_{0} \leqslant 5$.

In Figure 2 we show a plot of $0 / \mathrm{H}$ versus $\mathrm{N} / 0$ where, as in Figure 1 , the shaded area corresponds to PN of Type II; in this area the objects with lower $0 / \mathrm{H}$ and $\mathrm{N} / 0$ values are located in the outer regions of our galaxy, while the objects with higher $0 / H$ and $N / 0$ values are located 


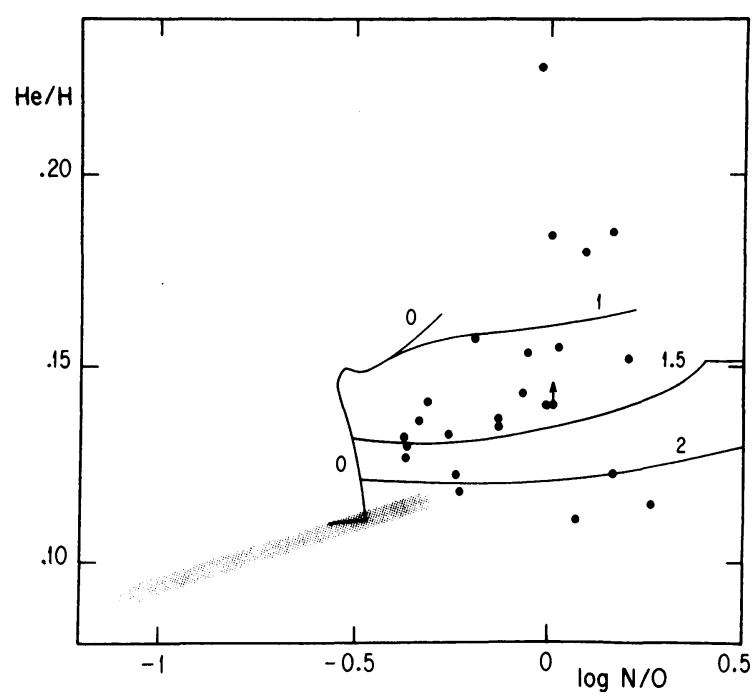

Fig. 1. Observed abundances of Planetary Nebulae. The shaded areas correspond to Type II objects, filled circles to Type I objects from Table 1. Stellar evolution predictions by Renzini and Voli (1981) are shown for different values of $\alpha$.

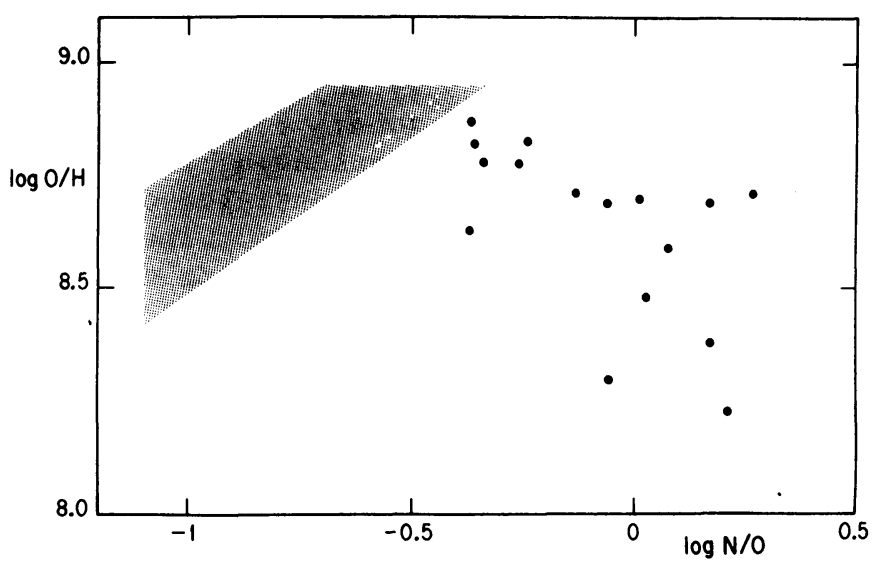

Fig. 2. Relative oxygen to nitrogen abundance. Filled circles correspond to Type I objects from Table 1, shaded area to Type II objects.

towards the center of our galaxy. The He and $\mathrm{N}$ rich objects are represented by filled circles, these objects seem to show a correlation in the sense that the higher the $\mathrm{N} / 0$ ratio the smaller the $0 / \mathrm{H}$ ratio; this trend was noticed before (Peimbert 1978; Kaler 1980) but no statistical significance was attached to it. From the work by Renzini and Voli (1981) it is found that hot-bottom burning depletes oxygen somewhat in highermass stars, however this effect seems to affect only slightly the surface 
abundances. There are two other possible explanations for the observed correlation in Figure 2: a) that some of the objects are of Population II or that the $0 / \mathrm{H}$ abundances are not reliable. The two objects with smallest $0 / \mathrm{H}$ abundance ratios are $\mathrm{Hu} 1-2$ and $\mathrm{Me} 2-2$; from the high radial velocity measurements it seems that Me 2-2 is a Population II object (PS) while recent observations by Torres-Peimbert et al. (1982) indicate that Hu 1-2 is not a high velocity object. With respect to the second possibi lity it should be noted that $\mathrm{Hu}$ 1-2 and Me 2-2 are the two objects with highest $N_{e}$ values observed by PTP and it is possible for a fraction of the $\mathrm{H}$ lines to originate in regions with $\mathrm{Ne}_{\mathrm{e}}^{>} 10^{6} \mathrm{~cm}^{-3}$ producing an underestimation of the real $0 / \mathrm{H}$ abundance ratio; this effect is present in CRL 618 and M 2-9 (Schmidt and Cohen 1981; Calvet and Peimbert 1982, hereinafter $\mathrm{CP}$ ). It is necessary to obtain accurate $0 / \mathrm{Ar}$ ratios because if $0 / \mathrm{Ar}$ is underabundant then it is likely that 0 has been depleted, on the other hand if $0 / \mathrm{Ar}$ is normal the other two possibilities are still open.

The C/O ratios of NGC 2440, NGC 6302 and NGC 6853 have been derived from UV data and are smaller than 1. There are two effects that can reduce the $\mathrm{C} / 0$ value as determined from $U V$ data: a) the use of a higher $\mathrm{T}_{e}$ than the real one when the 0 abundance is derived from optical lines, and $b$ ) the high optical thickness in the resonance 1548-1551 lines of C IV (e.g. Peña and Torres-Peimbert 1982). Under the assumption that $\mathrm{C} / 0=\mathrm{C}^{++} / \mathrm{O}^{++}$and from the $1909 / 1663$ intensity ratios for NGC 6302 by Aller et al. (1981) and Barral et al. (1982) we obtain $\log \mathrm{C} / 0=-0.35$; the $\mathrm{H}, \mathrm{He}, \mathrm{C}, \mathrm{N}, \mathrm{O}$ abundance ratios for this object are in agreement with models by Renzini and Voli (1981) with $M_{i} \sim 6 M_{\odot}$ and $\alpha=2$, NGC 6302 seems to be the most massive well documented object. It is also possible to derive $C$ abundances from the $\lambda 4267$ line of C II since apparently this line is produced by recombination only (see, Torres-Peimbert et al. 1980; Harrington et al. 1980). The problem with this method is that $\lambda 4267$ is very faint and often has been overestimated. Under the crude assumption that $\mathrm{C}^{+} / \mathrm{O}^{++}=\mathrm{C} / 0$ from the optical observations by PTP we obtain that for seven Type I PN $\log \langle\mathrm{C} / \mathrm{O}\rangle=+0.45$ which together with their $\mathrm{He} / \mathrm{H}$ values imply progenitors in the $3<M / M_{\odot}<5$ mass range with $2<\alpha<1.5$ (Renzini and Voli 1981).

\section{MORPHOLOGY}

Most of the objects presented in Table 1 are extremely filamentary and we have marked them with an F. B stands for binebulous, bipolar, biaxial or hourglass, the same object has been called in the literature by one or more of these adjectives (e.g. Westerlund and Henize 1967; Greig 1971; Phillips and Reay 1977; CP). V stands for a velocity field which is bipolar. S stands for relatively smooth and includes 5 objects which are not class B objects according to the classification by Greig; the abundances of these objects are not extremely He and $\mathrm{N}$ rich and in some cases are of low accuracy. C stands for compact, we do not have enough resolution to tell if these objects are filamentary or not. 
In the last column of Table 1 we present a list of representative references related to the morphological or internal velocity fields of these objects.

The majority of the objects in Table 1 are bipolar, several mechanisms have been proposed to explain bipolarity in PN, we will mention three of them. The three are based on the necessity of the ejection of a shell which is denser near the equatorial plane, due to gravitational braking, differential rotation and radiation pressure (e.g. Phillips and Reay 1977; Sabbadin and Hamzaoglu 1981; CP and references in these papers). CP argue that only stars with $M>2 M_{\odot}$, in the main sequence, have enough angular momentum to produce a shell significantly denser in the equatorial plane, they attribute the morphological difference between PN of Type I and others mainly to this fact. Phillips and Reay consider that the principal mechanism determining shell development is gravitational braking; Sabbadin and Hamzaoglu consider that the ansae are produced by Lyman continuum and Lyman $\alpha$ radiation pressure as well as thermal diffusion into vacuum; $C P$ consider that ejection takes place continuously with a velocity increase due to a decrease in the stellar radius and an increase in the escape velocity, two mass loss phases can be distin guished the first one mentioned above which is responsible for the tormation of a disc, and a second one which is responsible for the formation of the ansae. This second phase has been studied by Cantó (1980) for several astronomical scenarios; Meaburn and Walsh (1980) were the first to explain the bipolarity of the Type I PN NGC 6302 based on the model by Cantó (1980). The presence of a dense disc, which supports the suggestion by CP for the origin of bipolar PN, has been established for several objects (e.g. CP, Rodríguez and Morân 1982).

The filamentary structure of PN of Type I is probably due to shocks and instabilities produced by denser, and or, faster material catching up with material ejected previously. The filamentary structure is responsible for large variations in $\mathrm{Ne}_{e}$. The very hot central stars (see below) together with the density distribution are responsible for the extreme variations in the ionization degree of these objects.

From their bipolar and extremely filamentary structure the following objects are proposed as PN of Type I candidates: NGC 2899, NGC 3699, $\mathrm{Hb} 5$, He 2-29, He 2-76, He 2-111, He 2-114, He 2-207, K 3-46 and M 3-28 (see pictures in Westerlund and Henize 1967; and Perek and Kohoutek 1967). Webster (1978) has studied the velocity field of He 2-111, it resembles that of NGC 6302 , moreover the $6583 / \mathrm{H \alpha}$ ratio of He $2-111$ is of the order of 3 which probably indicates that it is $\mathrm{N}$ rich.

\section{MASSES}

In addition to the stellar evolution predictions based on chemical abundances that indicate $3 \leqslant M / M_{\odot} \leqslant 5$ for PN of Type $I$, there is additional evidence in favor of a relatively high mass for these objects. 
There are three PN of Type I for which a crude estimate of their masses at the zero age main sequence can be made: NGC 3132, NGC 2346 and NGC 2818. The average mass for these objects turns out to be $2.4 \mathrm{M}_{\odot}$ (PS; Méndez and Niemela 1981; CP). NGC 3132 and NGC 2346 are mild examples of He-N enrichment, therefore the lower mass limit for this process to occur would be around $2.4 \mathrm{M}_{\odot}$.

From the work by Greig (1972) based on the galactic kinematical properties of class B PN it follows that these objects are of Population I.

Kaler (1982a) has placed a few PN of Type I in the HR diagram, they tend to be at higher $\mathrm{T}^{*}$ and lower $\mathrm{L}^{*}$ with respect to other PN which, when compared with theoretical tracks (Paczyński 1971; Iben and Renzini 1982; Schunberner and Weidemann 1981), indicates that their core masses are higher than those of other PN nuclei.

\section{PROTOPLANETARY NEBULAE}

There are several bipolar nebulae that have been called protoplanetary nebulae in the literature, in general these objects have $\mathrm{T}^{*} \leqslant 35000^{\circ} \mathrm{K}$. If these objects are going to develop into Type I PN they should be He-N rich. At their stellar temperatures a substantial fraction of the He atoms is expected to be neutral (e.g. Peimbert et al. 1974) and it is very difficult to determine their $\mathrm{He} / \mathrm{H}$ abundance ratio. On the other hand, it is relatively easy to determine their $\mathrm{N} / 0$ abundance ratio since most of their $\mathrm{N}$ and $\mathrm{O}$ is singly ionized inside the $\mathrm{H}$ II zone. $\mathrm{CP}$ have determined that $\mathrm{CRL} 618$ and $\mathrm{Mz}-3$ are $\mathrm{N}$ rich (see Table 1), alternatively it seems that $M$ 2-9 is not. The N/0 ratio should be determined for other suspected protoplanetary nebulae with bipolar structure.

\section{CONCLUSIONS}

The majority of the PN of Type I are bipolar. An explanation in the sense that their bipolarity is due to the high angular momentum of their precursors has been advanced by $\mathrm{CP}$. There are several independent arguments that indicate that their masses are in the $2 \leqslant \mathrm{M} / \mathrm{M}_{0} \leqslant 6$ range, in particular their $\mathrm{H}, \mathrm{He}, \mathrm{C}, \mathrm{N}$ and 0 relative abundances. The $0 / \mathrm{Ar}$ ratio should be determined for these objects to study if there has been an oxygen depletion during the evolution of the progenitor star. About $10 \%$ to $30 \%$ of the PN are of Type I.

It is a pleasure to acknowledge L.H. Aller, I. Iben Jr., J.B. Kaler, S.R. Pottasch, A. Renzini and M.J. Seaton for sending us manuscripts in advance of publication. We are grateful to N. Calvet, J.F. Rayo and A. Serrano for several discussions and comments on this work. 
REFERENCES

Aller, L.H. and Czyzak, S.J.: 1978, Proc. Nat1. Acad. Sci. USA, 75,Pp.1-3. Aller, L.H. and Czyzak, S.J.: 1982, preprint.

Aller, L.H., Gzyzak, S.J., Craine, E., and Kaler, J.B.: 1973, Astrophys. J. 182, pp. 509-515.

Aller, L.H., Ross, J.E., O'Mara, B.J., and Keyes, C.D.: 1981, Monthly Notices Roy. Astron. Soc. 197, pp. 95-106.

Barker, T.: 1978, Astrophys. J. 220, pp. 193-209.

Barral, J.F., Cantô, J., Meaburn, J., and Walsh, J.R.: 1982, Monthly Notices Roy. Astron. Soc. 199, pp. 817-832.

Calvet, N., and Peimbert, M.: 1982, Rev. Mexicana Astron. Astrof., submitted.

Cantó, J.: 1980, Astron. Astrophys. 86, pp. 327-338.

Carsenty, U., and Solf, J.: 1982, Astron. Astrophys. 106, pp. 307-310.

Cohen, M., FitzGerald, M.P., Kunkel, W., Lasker, B.M., and Osmer, P.S.: 1978, Astron. J. 221, pp. 151-162.

Curtis, H.D.: 1918, Pub1. Lick Obs. 13, pp. 57-74.

Felli, M., and Perinotto, M.: 1979, Astron. Astrophys. 76, pp. 69-74.

Goudis, C., Mc Mullan, D., Meaburn, J., Tebbutt, N.J., and Terrett, D.L.: 1978, Monthly Notices Roy. Astron. Soc. 182, pp. 13-25.

Greig, W.E.: 1971, Astron. Astrophys. 10, pp. 161-174.

Greig, W.E.: 1972, Astron. Astrophys. 18, pp. 70-78.

Harrington, J.P., Lutz, J.H., Seaton, M.J., and Stickland, D.J.: 1980, Monthly Notices Roy. Astron. Soc. 191, pp. 13-22.

Iben $\mathrm{Jr} .$, I. and Renzini, A.: 1982, preprint.

Kaler, J.B.: 1978, Astrophys. J. 226, pp. 947-962.

Kaler, J.B.: 1979, Astrophys. J. 228, pp. 163-178.

Kaler, J.B.: 1980, Astrophys. J. 239, pp. 78-88.

Kaler, J.B.: 1982a, preprint.

Kaler, J.B.: 1982b, Astrophys. J. in press.

Meaburn, J., and Walsh, J.R.: 1980, Monthly Notices Roy. Astron. Soc. 191, pp. 5-11p.

Méndez, R.H., and Niemela, V.S.: 1981, Astrophys. J. 250, pp. 240-247.

Minkowski, R.: 1964, Publ. Astron. Soc. Pacific 76, pp. 197-209.

Paczyński, B.: 1971, Acta Astr. 21, pp. 417-435.

Peimbert, M.: 1978, in Y. Terzian (ed.), "Planetary Nebulae, IAU Symp. No. 76", Dordrecht: Reidel, p. 215.

Peimbert, M. : 1981, in R.D. Chapman (ed.), "The Universe at U1traviolet Wavelengths", NASA CP-2171, pp. 557-565.

Peimbert, M., Rodríguez, L.F., and Torres-Peimbert, S.: 1974, Rev. Mexicana Astron. Astrof. 1, 129-141.

Peimbert, M., and Serrano, A.: 1980, Rev. Mexicana Astron. Astrof. 5, pp. 9-18.

Peimbert, M., and Torres-Peimbert, S.: 1982, Rev. Mexicana Astron. Astrof. submitted.

Peña, M., and Torres-Peimbert, S.: 1982, Rev. Mexicana Astron. Astrof. submitted.

Perek, L., and Kohoutek, L.: 1967, "Catalogue of Galactic Planetary Nebulae", Academia Praha.

Phillips, J.P., and Reay, N.K.: 1977, Astron. Astrophys. 59, pp. 91-110. 
Pottasch, S.R.: 1981, private communication.

Renzini, A., and Voli, M.: 1981, Astron. Astrophys. 94, pp. 175-193. Rodríguez, L.F., and Morán, J.M.: 1982, Nature, submitted.

Sabbadin, F., and Hamzaoglu, E.: 1981a, Astron. Astrophys. 94, pp. 25-28. Sabbadin, F., and Hamzaoglu, E.: 1981b, Monthly Notices Roy. Astron. Soc. 197, pp. 363-368.

Schmidt, G.D., and Cohen, M.: 1981, Astrophys. J. 246, pp. 444-454.

Schönberner, D., and Weidemann, V.: 1981, in I. Iben Jr. and A. Renzini (eds.) "Physical Processes in Red Giants", Dordrecht: Reidel, pp. 463-468.

Serrano, A., and Peimbert, M.: 1981, Rev. Mexicana Astron. Astrof. 5, pp. 109-124.

Shields, G.A., Aller, L.H., Keyes, C.D., and Czyzak, S.J.: 1981, Astrophys. J. 248, pp. 569-583.

Torres-Peimbert, S., and Peimbert, M.: 1977, Rev. Mexicana Astron. Astrof. 2, pp. 181-207.

Torres-Peimbert, S., Peimbert, M., and Daltabuit, E.: 1980, Astrophys. J. 238, pp. 133-139.

Torres-Peimbert, S., Peimbert, M., Rayo, J.F., and Peña, M.: 1982, Rev. Mexicana Astron. Astrof. submitted.

Webster, L.B.: 1978, Monthly Notices Roy. Astron. Soc. 185, pp. 45-50p. Westerlund, B.E., and Henize, K.G.: 1967, Astrophys. J. Supp1. 14, Pp. 154-169.

Worswick, S.P.: 1980, private communication.

DOPITA: Many of your type I candidates have been confirmed by

(unpublished) observations of Louise Turtle (nêe Webster) and myself.

ACKER: I studied relations between chemical, spatial and kinematic properties of $97 \mathrm{PN}$. Those with high $\mathrm{He} / \mathrm{H}$ and $\mathrm{N} / \mathrm{O}$ enrichment (most of which are of morphological type "B"; their nuclei have continuous or type 0 spectra and their mean excitation class is high) have a low mean distance from the Galactic plane $(\langle|z|\rangle=210 \mathrm{pc})$ and a very flat velocity ellipsoid. Their spatial and kinematic parameters correspond to an initial mass of about $3 \mathrm{M}$.

WEIDEMANN: Concerning secondary Criteria: what fraction of the central stars are visible?

PEIMBERT: We are just in the process of looking into this problem. For a substantial fraction of these objects, we do not see the central star, but many of them are very far away and we need a good distance to obtain a meaningful upper limit in the H-R diagram.

KALER: Returning to the $\log (\mathrm{N} / \mathrm{O})$ - He/H correlation, you obtain a better fit if you adopt the Becker and Iben calculation with partial conversion of $C$ to $\mathrm{N}$.

NUSSBAUMER: I recently suggested that acceleration by emerging magnetic flux might be a mechanism producing PN (Astron. Astrophys. 110, L1). Bipolar nebulae might be good candidates for having been produced through magnetic activity in open polar field structures. 
TERZIAN: Why did you not include NGC 7027 in PN of type I, since its radio morphology is bipolar and its central mass is estimated to be about $1 \mathrm{M}$ ? If $\mathrm{I}$ may make a more general comment on PN morphology, although one PN looks different from another, there are some overall similarities and bipolarity is one of these. Almost all high resolution radio maps show some degree of intensity bipolarity.

PEIMBERT: NGC 7027 does not quite meet the $\mathrm{He}-$ and N-rich criterion for type I PN. Furthermore, NGC 7027 belongs to those objects which exhibit "very mild" bipolarity. Extreme cases of bipolarity are observed: a wel1 defined disk with ansae present (e.g. NGC 6302, NGC 2440, Mz-3, and He 2-111); weak disk confinement and absence of ansae (e.g. NGC 2474-5). 